\title{
On Routing with QOS Constraints in ATM Networks
}

\author{
Dirceu Cavendish and Mario Gerla \\ University of California at Los Angeles \\ Computer Science Dept., School of Engineering and Applied Science, \\ 405 Hilgard Avenue, Los Algeles, CA, 90024, USA. \\ Telephone: 1310 8254367. Fax: 13108257578. \\ email: $\{$ dirceu,gerla\}@cs.ucla.edu
}

\begin{abstract}
Multimedia applications require guaranteed QOS, which will be provided by multimedia networks, such as ATM, by resource reservation. The complexity of routing and allocating resources for ATM applications vary according with applications end-to-end constraints, and essentially range from P to NP complete problems (Wang et al (1995)). In this paper, we expand the class of known $\mathrm{P}$ type, constrained routing problems, by including two applications of significance in ATM, namely loss sensitive ABR traffic, and delay sensitive VBR traffic. For such routing problems, we present a $O\left(N^{4}\right)$ polynomial time algorithm, prove its correctness, and illustrate its effectiveness with simulation experiments.
\end{abstract}

Keywords

ATM Routing, End-to-end Flow Control, Quality of Service Constraints

\section{INTRODUCTION}

Broadband networks based on Asynchronous Transfer Mode (ATM) are being designed for the integration of various types of traffic, such as voice, data, and video. These networks are virtual circuit oriented, and they must provide end-to-end Quality Of Service (QOS) guarantees to the connections, which may vary from application to application.

The goal of providing end-to-end guarantees has a considerable impact on the design of algorithms at various protocol levels of the network. To mention a few, intermediate switches must provide bounds on service delays and loss; Call Admission Control (CAC) must take into account the available trunk bandwidth resources so that the end-to-end requirements of existing connections be maintained; Routing algorithms must provide routes which conforms with users end-to-end requirements while minimizing the cost of a connection. The design of efficient scheduling algorithms for meeting QOS requirements is an active research topic (Georgiades et al (1996), Ling et al (1996), Parekh et al (1993, 1994)). 
Routing with bandwidth allocation has been considered in Wand et al (1995), and is essentially a polynomial solvable problem with the same time complexity as Dijkstra algorithm. Routing problems which consider pairs of link costs of additive nature, such as delay and number of hops, have been studied as restricted shortest path problems, as in Hassin (1992), and are known to be NP-complete problems. The complexity of these problems depends heavily on the definition of link costs and end-to-end requirements.

In ATM nomenclature, links can be associated with two types of parameters (PNNI Draft Specification (1994)), link metrics and link attributes. Link attributes are parameters which can be consulted in isolation when deciding the link eligibility for carrying a connection. Bandwidth is an example of a link attribute. Link metrics, on the other hand, are of additive nature, hence they need to be considered in conjunction with other links in a given path in order to decide path eligibility for carrying a QOS constrained connection. Delay is an example of a link metric. When link attributes only are involved, the problem has been shown to be easily solvable (Wang et al (1995)). For instance, the available bandwidth of a path is the minimum bandwidth available among all its links. However, for routing problems involving multiple link metrics, such as delay and network operation cost (called administrative cost in PNNI Draft Specification (1994)), the problem becomes NP-complete.

This paper identifies, for the first time, a new class of polynomially solvable routing problems, which in terms of complexity lies between routing problems considered by Wang et al (1995) and Hassin (1992). We argue that the task of routing some ATM applications can be modeled by this class of problems.

The paper is organized as follows. Section 2 defines precisely the routing problems of our interest, including the class introduced in this paper. In section 3 , we present an algorithm for routing this new class of problems, based on executing rounds of the Dijkstra shortest path algorithm, and prove its correctness. Section 4 identifies ATM applications for which our routing algorithm is suitable. In section 5 we describe a network topology used to evaluate routing for some of the proposed applications. We present simulation results, comparing call acceptance probability versus network load for these applications. Conclusions are drawn in the last section. An Appendix is included with pseudo code of the routing algorithms used in the simulation experiments.

\section{ROUTING PROBLEM DEFINITION}

As mentioned earlier, multimedia networks will require routing algorithms which are capable of dealing with multiple link metrics. In this section we start by describing a NPcomplete routing problem precisely. Then we define a routing problem of lesser complexity.

We assume that each link has only two independent metrics. We model a multimedia network as a generic graph, in which paths must be established between nodes so that path constraints be met. Thus, a graph $G=(V, E)$ consists of a set $V$ of vertices and a set $E$ of edges. Associated with each edge $e \in E$ is a length $l(e) \in R^{+}$and a weight $w(e) \in R^{+}$. A path $p$ is a sequence of distinct vertices $v_{1}, v_{2}, \cdots, v_{k}$ such that $\left(v_{i}, v_{i+1}\right) \in E$ for $i=1, \cdots, k-1$. The set $\left(v_{i}, v_{i+1}\right)$ is called the edges of $p$. The length (weight) of $p$, $L(p)(W(p))$ is the sum of the lengths (weights) of the edges of $p$.

The NP-complete problem is defined as follows: find a minimum length path connecting two specific vertices $(\mathrm{s}, \mathrm{d})$, while satisfying a path weight bound $\mathrm{W}$. This routing problem 
can be formulated by the following optimization problem: Minimizing the cost function $l(p)$ of a path $p$ between a specific source/destination pair $(\mathrm{s}, \mathrm{d})$, where

$l(p)= \begin{cases}\sum_{i \in p} l(i) & \text { if } \sum_{i \in p} w(i)<W \\ \infty & \text { otherwise }\end{cases}$

As mentioned earlier, this routing problem has been studied in Hassin (1992). We now define a new routing problem. Let the cost of a path be defined slightly differently, as follows:

$l(p)= \begin{cases}\sum l\left(v_{i}, v_{i+1}\right) & \text { if for each edge }\left(v_{i}, v_{i+1}\right), w\left(v_{i}, v_{i+1}\right)>M \sum l\left(v_{i}, v_{i+1}\right) \\ \infty & \text { otherwise }\end{cases}$

where $M$ is a constant. Notice that in this definition the second link parameter $w$ can be considered a link attribute, in a sense that one does not need the values of other $w$ links parameters to decide link eligibility. However, one still needs to consult the $l$ metric values of the other links to decide eligibility. We define the shortest path length from $s$ to $d$ to be:

$\delta(s, d)= \begin{cases}\min [l(p): s \leadsto d\} & \text { if } \exists \text { a path from } s \text { to } d \\ \infty & \text { otherwise }\end{cases}$

In the next section, we present a routing algorithm which finds shortest paths according to this last definition between any two vertices of a graph.

\section{BOTTLENECK DISCARDING ROUTING PRINCIPLE}

The main routing strategy is rather straightforward. We use a Dijkstra algorithm to find the shortest path between a given pair $(\mathrm{s}, \mathrm{d})$ of vertices. However, at each step of the algorithm, when adding new edges, we verify that the cumulative path weight does not violate the weight constraint defined in Eq. (1), which would raise the cost of the path to infinity. If the constraint is violated, the bottleneck link, which is defined to be the link with highest weight value so far, is discarded, and a new attempt to find a feasible path starts. We define a phase of the algorithm to be the interval from when the algorithm starts path computation until it either finishes the computation or it detects constraint violation. The algorithm halts when either it finds a path or the source gets disconnected, in which case no path is found. We name this routing strategy the bottleneck Dijkstra algorithm, B-Dijkstra. A B-Dijkstra pseudo-code follows. 


\subsection{B-Dijkstra Pseudo-code}

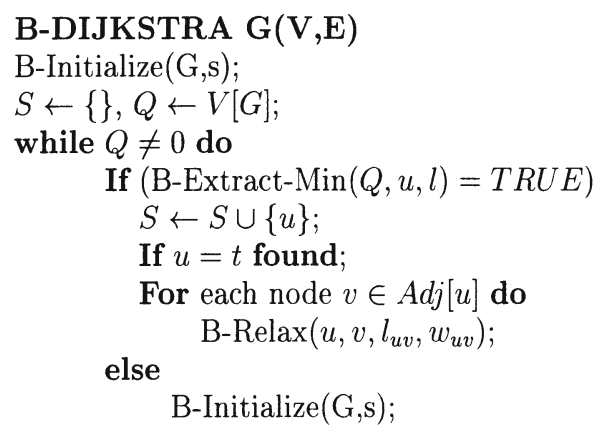

where the subroutines B-Initialize $(\mathrm{G}, \mathrm{s}), \mathrm{B}-\operatorname{Extract-Min}(\mathrm{Q}, \mathrm{u}, \mathrm{l})$ and B-Relax $\left(u, v, l_{u v}, w_{u v}\right)$ are described below:

\section{B-Initialize(G,s)}

For each node $v \in V[G]$

Do $l_{v} \leftarrow \infty ; w_{v} \leftarrow \infty ; \pi_{v}=N I L$

$l_{s} \leftarrow 0 ; w_{s} \leftarrow 0$;

$w_{\text {max }}^{i}=\infty ; b l^{i}=N I L$;

\section{B-Extract-Min $(\mathrm{Q}, \mathrm{u}, \mathrm{l})$}

$d_{\min } \leftarrow \infty ; u \leftarrow N A N$;

For $i \in Q$ do

$$
\begin{aligned}
\text { If } & \left(l_{i}<d_{\min }\right) \\
& \left\{d_{\min } \leftarrow l_{i} ;\right\}
\end{aligned}
$$$$
u \leftarrow i \text {; }
$$

If $\left(w_{u}<W_{s t}\right)$

RETURN TRUE;

else

$l_{* \pi\left(b l^{u}\right), b l^{u}} \leftarrow \infty$

RETURN FALSE;

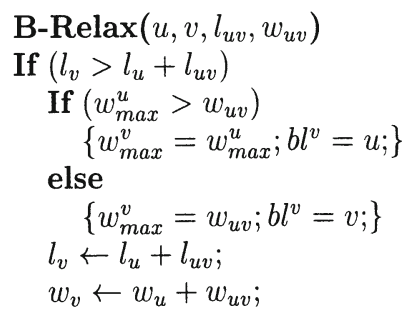

$\mathrm{S}$ is a set of nodes whose current shortest path is maintained, and $\mathrm{Q}$ is a priority queue with nodes in $\mathrm{V}-\mathrm{S}$ with their current distances $l_{i}$. Each node $u$ has a pointer $\pi_{u}$ to its 
previous node in the current shortest path, which is initially set to NIL. B-Extract-Min fetches the node outside $\mathrm{S}$ which is closest to the source, and B-Relax updates nodes distances to the shortest ones, as is standard in Dijkstra's algorithm. The twist here is that, by keeping track of the path weight $w(p)$, B-Extract-Min routine checks if the path weight constraint is violated. If this happens, the bottleneck link (in weight) is discarded and the algorithm restarts. Since for each Dijkstra phase of the algorithm, at worst the link next to the source is discarded when we are next to reaching the destination $t$, we have $|E|$ phases of the usual Dijkstra algorithm at the most. Each Dijkstra phase taking $O\left(N^{2}\right)$ time (Cormen et al (1990)), for a complete graph we obtain $O\left(N^{4}\right)$ complexity for B-Dijkstra. Although this time seems to be still excessive for large networks, simulation experiments reveal that the average running time of B-Dijkstra is very close to the original Dijkstra, mainly because we are likely to discard only few links in the computation of a path. In the following subsection, we prove the algorithm correctness.

\subsection{B-Dijkstra Correctness Proof}

We now prove B-Dijkstra correctness. More formally:

Theorem 1 B-Dijkstra algorithm computes the $(s, t)$ shortest path as defined in definitions 2 and 1.

To prove the theorem, we first need the following lemma:

Lemma 1 Consider a generic node $y$ at an intermediate step of the $s, t$ shortest path computation. Then, $l(s, y)=\delta(s, y)$ when node $y$ is inserted into set $S$, after being chosen by B-Extract-Min.

Proof. The proof is identical to the equivalent claim for DIJKSTRA algorithm, which can be found in Cormen et al (1990), for example. The additional path constraint does not invalidate the proof there described.

Lemma 1 guarantees that, as long as edges are not discarded, the shortest distance from $s$ to any destination is obtained when the algorithm halts. Next, it remains to be proven that the discarding of the bottleneck edge does not invalidate the lemma.

Figure 1 depicts a typical step of the algorithm. Let node $u$ be the node fetched by B-Extract-Min, when computing the optimal path from source $s$ to destination $t$. Let also edge $(m, n)$ be the bottleneck edge of the path $(s, u)$. Moreover, define the bottleneck resource $w^{b}(u)$ as the minimum value $w\left(v_{i}, v_{i+1}\right)$ along the path $(s, u)$. 


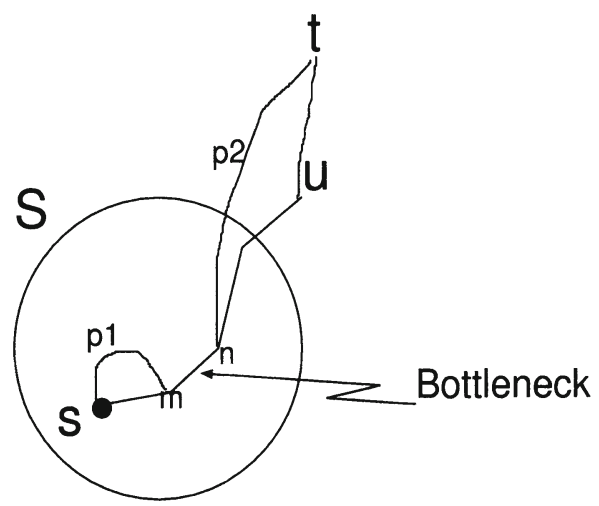

Figure 1 B-Dijkstra correctness proof

Proof. For each node $u$ fetched by B-Extract-Min, $u \in Q$, and $\delta_{u}=\min _{i \in Q}\left[l_{i}\right]$, two cases are possible:

i) $w^{b}(u)>M \sum_{p} l\left(v_{i}, v_{i+1}\right):$ In this case, lemma 1 guarantees minimum distance computation.

ii) $w^{b}(u)<M \sum_{p} l\left(v_{i}, v_{i+1}\right):$ In this case, the bottleneck edge is eliminated. We must show that no feasible path containing such bottleneck exists. Lets suppose there is a feasible path $(s, p 1, m, n, p 2)$. Notice that edge $(m, n)$ may or may not be the bottleneck of this path. Let the new path bottleneck value at $t$ be $w^{b^{\prime}}(t)$. We know that $w^{b}(u)<M l(s, u)$. By using lemma 1 for nodes $m$ and $u$, we know that $l(p 1) \geq \delta(s, m)$, and thus the path $(s, p 1, m, n, p 2)$ is longer than $l(s, u)=\delta(s, u)$. If the bottleneck edge for path $(p 1, n)$ is $(m, n)$, the previous observation that the alternate path has longer length than the original one leads to the conclusion that the path constraint is also violated for the alternate path. If the bottleneck of path $(p 1, n)$ is not $(m, n), w^{b^{\prime}}(n)$ has an even smaller value than the original bottleneck $w^{b}(t)$, which causes the path constraint to be violated as well. Therefore, there is no feasible alternate path that includes bottleneck $(m, n)$.

\section{ROUTING ATM TRAFFIC WITH B-DIJKSTRA}

In this section, we describe various ATM networks applications in which the B-Dijkstra can be effectively used as a routing algorithm. First we review the key network assumptions. ATM switches are output buffered. The resources to be allocated in the switches in order to guarantee end-to-end requirements are buffer space and service rate (bandwidth). Although commercial ATM switches may not allow buffer allocation, recent research papers indicate that buffer allocation is necessary for QOS control (Georgiadis et al (1996), Mascolo et al (1996)). Each switch has a dedicated buffer space per output port (although this assumption can be relaxed). We assume route computation at the source (as per link state, or OSPF routing). Available link resources (buffer, bandwidth) are broadcasted at 
regular intervals to each ATM node, where a topology map of the network is kept. In this map, each link $(i, j)$ is associated with a buffer size $B_{i j}$ and a Capacity (bandwidth) $C_{i j}$. Resources are reserved in a FCFS fashion at call request time. If there are $N_{i j}$ connections sharing link $(i, j)$, where connection $k$ has $\left(b_{i j}^{k}, c_{i j}^{k}\right)$ buffer and capacity resources allocated, respectively, the broadcast information for that link is given by:

$\begin{array}{ll}\text { available capacity: } & c_{i j}^{a v}=C_{i j}-\sum_{1}^{N_{i j}} c_{i j}^{k} \\ \text { available buffer: } & b_{i j}^{a v}=B_{i j}-\sum_{1}^{N_{i j}} b_{i j}^{k}\end{array}$

We do not consider failures of any type (neither link nor switch) in this paper.

\subsection{Routing Loss sensitive ABR traffic}

Loss sensitive ABR traffic is a data application characterized by some minimum bandwidth requirement, MCR. The ATM Forum, in its PNNI Draft Specification (1994), allows for loss sensitive ABR traffic. TCP traffic carried through an ATM network is an example, where cell loss triggers retransmission at TCP level, leading to throughput degradation. Traffic shaping may be performed at the network entry point to regulate source transmission rate. Within the network, buffers as well as bandwidth must be reserved to keep cell loss at an acceptable level.

For this type of application, the sustainable input rate is clearly related to buffers available at each link traversed by that connection, level of acceptable loss, and feedback loop delay, which in turn depends on the chosen path. For a connection $(s, t)$ requesting $\left(b_{s t}, c_{s t}\right)$ resources, the routing algorithm must find the minhop path which includes only links $(i, j)$ such that $c_{i j}^{a v}>c_{s t}$ and $b_{i j}^{a v}>b_{s t}$. To complicate matters, however, while $c_{s t}$ comes directly from traffic declaration, $b_{s t}$ is likely to depend upon the round trip delay of the path yet to be computed, since the performance of the congestion control mechanism is affected by the round trip delay. For example, if the rate controller described in Mascolo et al (1996) is used, and zero cell loss is required, the buffer $b_{s t}$ to be reserved for a connection $(s, t)$ is:

$b_{s t}=c_{\text {stmax }}\left(\frac{1}{K_{c}}+R T D\right)$

where $K_{c}>0$ is the feedback control gain, $c_{\text {stmax }}$ is the maximum input rate of connection $(s, t)$, and RTD is the round trip delay of the connection. This simply states that we need buffers of the order of the number of cells circulating inside the feedback loop. For sake of generality, by choosing the fastest rate controller $\left(K_{c}=\infty\right)$, we adopt the simplified relation:

$b_{s t}=K c_{\text {stmax }} R T D$

where $\mathrm{K}$ is a proportionality constant to be tuned according to the acceptable cell loss level. We conjecture that this relation must hold regardless of the rate control scheme 
used, as long as the controller attempts to set a limit on cell loss, and therefore takes into account the round trip delay. Of course the minimum RTD path exhibits the smallest buffer requirement. A simple approach would be to use a Dijkstra algorithm to compute the minimum RTD path, and then check if the following constraint is satisfied for each link $(i, j)$ along the chosen path:

$b_{i j}>K c_{s t \max } R T D$

However, if repeated call requests occur between a given source/destination pair, this approach would eventually lead to call rejection due to exaustion of buffer resources, even though an alternate path with large buffer space might exist.

Performance can be improved using a QOS routing algorithm as follows. Let us define the propagation delay of link $i, p d_{i j}$, in terms of the number of inflight cells in the link (i.e $p d_{i j}=$ prop.delay $y_{i j} \times c_{i j}$. Furthermore, since for ABR connection $(s, t)$ only the minimum rate $c_{s t}$ must be guaranteed by the network, we rewrite equation (3) as $b_{s t}=K c_{s t} R T D$ by adjusting the gain $K$ accordingly. To support the feedback control, we assume that the links $(i, j)$ are bidirectional, i.e. $p d_{i j}=p d_{j i}$. The pseudo-code of the algorithm for finding a path for $\mathrm{ABR}$ connection $(s, t)$ with minimum rate $c_{s t}$ is provided in the Appendix.

The ABR-DIJKSTRA algorithm follows the same relaxation principle as B-Dijkstra, with bottleneck discarding strategy. The minor differences are: At initialization, ABRDijkstra first discards all links which do not have the minimum required capacity. It also sets the available buffer space for a link to the minimum between the forward and reverse directions, since buffers are reserved in both directions to establish the feedback loop. For each node $v$, there is a variable $b_{\text {min }}^{v}$ that records the minimum buffer space available among all nodes in the shortest path from $s$ to $v$. Initially, $b_{\min }^{v} \leftarrow \infty$. There is also the variable $b n k$ which keeps track of the location where such minimum occurs (i.e. the bottleneck link). This way we do not have to recheck equation (4) for each node every time a path distance is changed.

\subsection{Routing VBR traffic with bounded delays}

The most common example of VBR traffic is compressed video, for which bounds on endto-end delays must be guaranteed. Video applications are expected to specify burstiness and average bandwidth. In turn, switches must allocate bandwidth and buffers necessary to cope with the burstiness and bandwidth declared, delivering the required delay performance (Georgiades et al (1996), Parekh et al (1993,1994)).

VBR routing requires the computation of bounds on end-to-end delay for all feasible paths. Among these, the one with minimum administrative path cost should be elected as the preferred one, in order to maximize network resource utilization. The end-to-end delay consists of two components: propagation delay; switching and scheduling delay. It turns out that propagation delay is additive and renders the problem NP complete. Switching and scheduling delays are also additive, but can be negotiated on a per link basis (as shown below), and therefore can be handled with a $\mathrm{P}$ problem formulation of the type shown in Eq. (1). In our approach, we ignore link propagation delays, assuming them negligible in face of switching and scheduling delays. In case propagation delays are significant, the bottleneck discarding strategy could be used for path computation in this 
case also, although there is no guarantee that the resulting path is optimum. In any event, a routing strategy which considers both types of link delays should take into account the delay negotiation presented in this section.

Scheduling disciplines have been proposed recently for providing switch delay bounds for multimedia connections (Georgiades et al $(1996)$, Parekh et al $(1993,1994)$ ). We use the work of Georgiades et al (1996) to express the dependencies between end-to-end delay bounds, bandwidth, burstiness, and link buffer and bandwidth allocation. In their work, end-to-end delay bounds are guaranteed through hop-by-hop traffic shaping, so that traffic burstiness be kept on the same levels throughout the connection path. For a call $(s, t)$ requiring end-to-end delay bound of $W_{s t}$, given its source traffic shaper, which can be a leaky bucket with parameters $(\sigma, \rho)$, we can compute the maximum delay $w_{i j}$ incurred at a link $(i, j)$, with rate and buffer resources $c_{i j}^{a v}, b_{i j}^{a v}$ respectively. Notice that $\sigma$ specifies the maximum burtiness of the VBR connection, whereas $\rho$ represents its average bit rate. More precisely, traffic shaping and scheduling algorithms will guarantee bounds on waiting and service delays provided that for output link $(i, j)$ we have $b_{i j}^{a v}>\sigma, c_{i j}^{a v}>\rho$. Namely, using a scheduling policy, such as NPEDF, the switching and scheduling delay bound $w_{i j}$ can be specified as:

$w_{i j}=\frac{\sigma}{\rho}+\frac{\sigma}{c_{i j}}$

where $c_{i j}$ is the total capacity of output link $(i, j)$. Disregarding propagation delays, a bound on end-to-end delay is obtained as follows:

$w_{s t}=\sum_{1}^{M} w_{i j}$

For networks with non uniform link capacities, equation (5) tells us that different switches offer different delay bounds. For now let us assume a uniform link speed $c$. Thus, the second factor of equation (5) is the same on all links of a given connection, and its end-to-end delay contribution is minimized by choosing the minimum hop path. Notice that link delay $w_{i j}$ can be reduced for each node along the path by allocating bandwidth $\rho^{\prime}$ larger than the declared rate $\rho$.

We summarize these observations in the following way: given a VBR connection $(s, t)$ with parameters $(\sigma, \rho)$ and end-to-end delay bound $W_{s t}$, a path $p$ with $M$ hops is said to be eligible if we can reserve for each link $(i, j), b_{i j}=\sigma$, and $c_{i j}=\rho$. Moreover, if $\sum_{1}^{M} w_{i j}<W_{s t}$ we are done. Otherwise, we must renegotiate the bandwidth $\rho^{\prime}>\rho$ so that we reduce the end-to-end delay to the required level. In this case, from Eq. (5), we have:

$\rho^{\prime}>\frac{M \sigma}{W_{s t}+\frac{M \sigma}{c}} \approx \frac{M \sigma}{W_{s t}}$

The approximation made in the last equation is used to argue in favor of using the bottleneck discard strategy only. Namely, Eq. (7) resembles equation (4), which states 
buffer requirements for ABR traffic. If RTD is measured in number of hops, these two relations are identical. The path delay, however, is still computed using the exact equation. The pseudo code for the routing strategy is presented in the Appendix.

The algorithm computes the minimum hop path from $s$ to $t$, checking at each step (when a new link is added) if equation (7) is satisfied. To do that, similarly to the ABR traffic algorithm, the position of the bottleneck link is tracked. If the minimum hop path can not satisfy equation (7), the bottleneck link is discarded, and a new instance of the algorithm is started.

Notice that the proposed algorithm will compute a minimum hop path which provides the tighest lower bound on the end-to-end delay required by the connection. This way, it is reasonable to expect that network resources are maximized, which leads to high overall network throughput.

\section{SIMULATION EXPERIMENTS}

In this section, we illustrate how the bottleneck based Dijkstra routing algorithm provides better network utilization by comparing it with the usual shortest path (minimum hop) and a maximum capacity algorithms. Our figure of merit here is the call acceptance probability for a given network load.

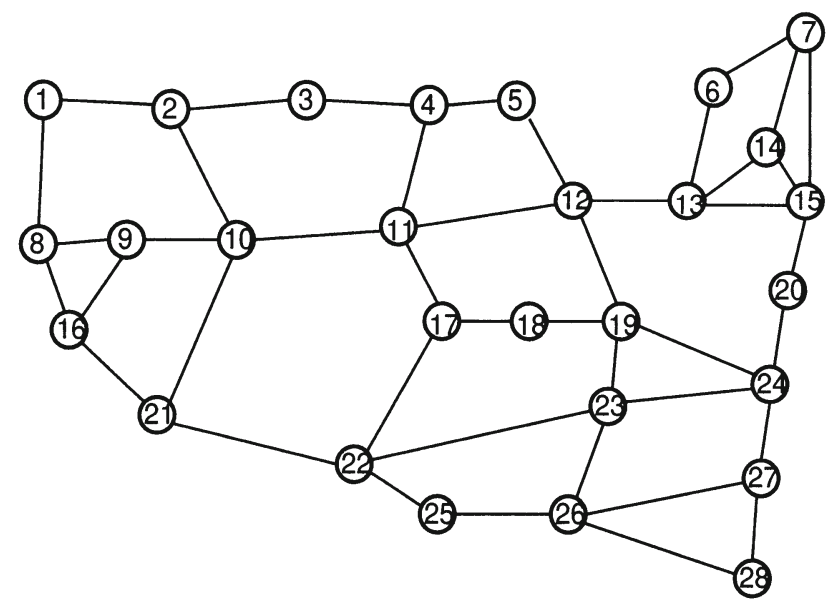

Figure 2 Network topology

We have simulated at call level a continental US size network. Figure 2 shows the network topology used in our experiments. The network diameter is 7 , and the average node degree is 3.07 . Link bandwidth is set to $155 \mathrm{Mb} / \mathrm{s}$. Buffers are provided on a per output link basis, with a uniform size of $155 \mathrm{Mbits}$, or approximately $365 \mathrm{~K}$ ATM cells. Since the network is of a small size, hierarchical routing, as specified by PNNI Draft Specification (1994), is not necessary. However, the routing strategy can be applied to each level of a more general hierarchical routing algorithm. A flooding scheme is used to 
convey link state information to all nodes. However, link state messages are generated in a periodic fashion only, not on an event driven basis. This is so because generally the effectiveness of event driven link state dissemination depends on the so called significant change events in PNNI nomenclature, or events that change network topology, such as link failures and the like. Since we do not include events of such nature, a periodic flooding strategy suffices. A new flooding procedure is initiated approximately every $50 \mathrm{~ms}$, so that source nodes have an accurate view of the network state. Call arrivals are Poisson distributed. Each source randomly selects a destination to place a call to, and uses a specific routing algorithm to compute the path. If no path is found, the call is rejected at the source. Otherwise, a call request is placed, which can be followed by a call acceptance, in case the appropriate resources are available, or call rejection, in case the resources are no longer available (i.e. the state of the resources along the path has changed with respect to the information on which call acceptance at the source was based upon).

\section{Switching delays case study}

In this set of simulation experiments, calls are placed at a rate of 200 per second per node. A VBR call is characterized by: burst duration of $70 \mathrm{msec}(\sigma=165$ cells), average rate $\rho=10 \mathrm{Mb} / \mathrm{s}$, and maximum end-to-end delay (excluding propagation delays) $W=$ $35 \mathrm{msec}$. Three routing strategies are compared: pure minhop Dijkstra routing; maximum capacity Dijkstra routing; and VBRSW-Dijkstra routing. The results are shown in Figure 3 .

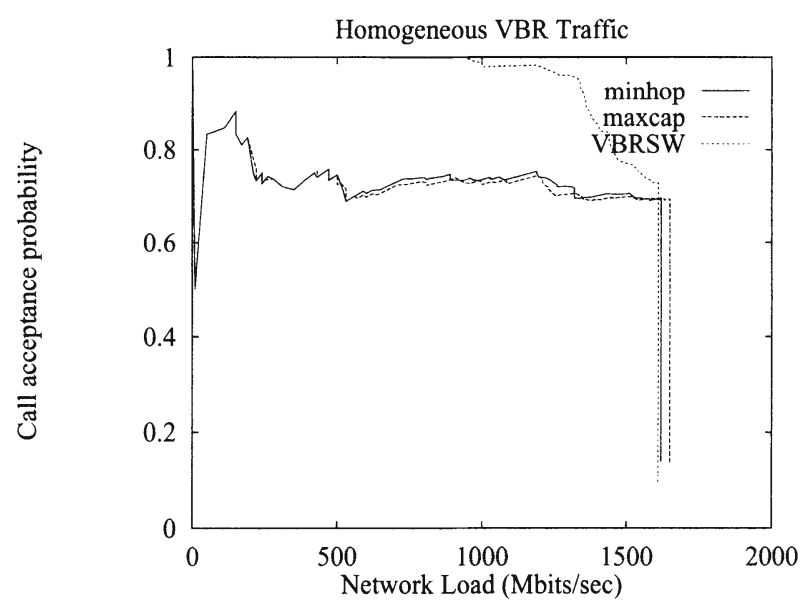

Figure 3 Call acceptance probability for homogeneous VBR traffic with switching and scheduling delays only

As expected, VBRSW-Dijkstra outperforms both maximum available capacity and minimum hop routing over the entire feasible network load range, up to saturation. Surprisingly, the two other schemes lead to similar call acceptance results. However, the exami- 
nation of simulation traces of the routing process shows that, although both algorithms exhibit similar behavior, they differ greatly in the routes computed. When the network is lightly loaded, both algorithms choose the same paths, since the available capacity is the same for all links. However, as network load grows, the minhop strategy tends to select short but saturated paths, which lead to call rejection due to end-to-end constraint violation. In turn, the maximum capacity algorithm tends to select less congested, but longer, paths, which also cause end-to-end constraint violation. VBRSW-Disjktra performs best among the three for all network loads because it keeps track of the minimum hop routing strategy among all feasible paths in terms of end-to-end delay requirement. Thus, it always provides the shortest possible path on which the delay QOS requirement is met. Furthermore, traces have shown that all successful paths found by minhop are also found by VBRSW-Dijkstra. The latter finds some additional (longer) ones, not explored by the minhop algorithm. By exploring alternative paths, VBRSW-Dijkstra is able to accomodate more calls over a broad network load range. We find that bottleneck link discarding happens only once or twice during a path computation, and hence the average running time of VBRSW-Dijkstra is close to the original Dijkstra algorithm, $O\left(N^{2}\right)$. This applies to other experiments, as well. Of course we should expect this number to increase for dense networks.

\section{Rate Controlled ABR case study}

Here we assume a network with available capacity for $\mathrm{ABR}$ traffic equal to $50 \mathrm{Mb} / \mathrm{sec}$ on each trunk. Call arrival process is still Poisson, with an average of 20 calls/sec per network node. Each call requires $1 \mathrm{Mb} / \mathrm{sec}$ rate, and zero cell loss. Thus, a feasible path must satisfy the buffer requirement $b_{i j}>c_{\text {stmax }} R T D$ for each link $(i, j)$ along the path. Figure 4 shows the results of minhop, maxcap, and ABR-Dijkstra routing algorithms.

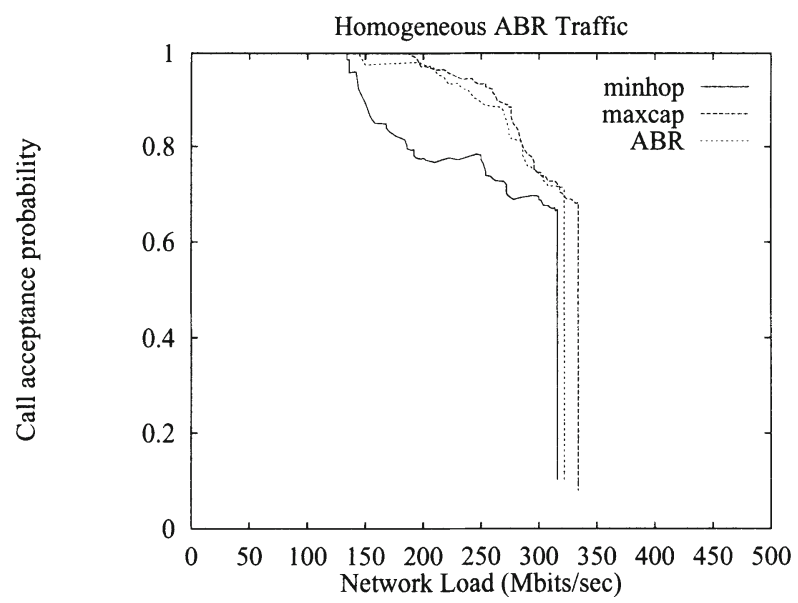

Figure 4 Call acceptance probability for homogeneous ABR traffic 
We see that pure minhop routing performs poorly. An interesting and rather surprising result is that in this case maximum capacity routing and ABR-Dijkstra routing perform similarly. In fact, maxcap seems to even outperform ABR-Dijkstra. This is surprising because ABR-Dijkstra was proven optimal in this problem, and hence should be able to find a feasible path whenever maxcap finds one. Therefore, ABR-Dijkstra call acceptance should be at least as high as for maxcap. The explanation of this apparent discrepancy is based on two facts. First, for our particular set of parameters, buffer availability is closely coupled with available link capacity, so that the buffer constraint is automatically satisfied by the maximum capacity routing strategy. In fact, calls are uniformly placed throughout the network, and hence the higher the load is, the lower the buffer space. Thus, the maximum capacity path is very likely to satisfy also the buffer constraint, since link delays are small, and hence few extra links on a path do not impact significantly on buffer requirement. Notice that, although the end-to-end constraint here is similar to the one tackled by VBRSW-Dijkstra, in the previous case few extra links would easily violate the end-to-end constraint, yielding to poor maxcap performance. Secondly, the route computation is based on link state information which is outdated with respect to the actual state of the network resources found during call set up time ${ }^{*}$. Notice that the ABR-Dijkstra solution is generally "tight", i.e. it has little slack in terms of buffers and bandwidth. Thus, a minor change in link resources along the chosen path may render the path unfeasible. The maxcap path, on the other hand, is more robust to such changes, since it has the path with the largest possible buffer/bandwidth slack.

\section{CONCLUSION AND FUTURE WORK}

We have proposed a new polynomial routing algorithm, called B-Dijkstra (BottleneckDijkstra), for ATM routing with path constraints. Although the worst case analysis of B-Dijkstra shows $O\left(N^{4}\right)$ complexity, simulations have shown that in practice the average running time of B-Dijkstra is close to $O\left(N^{2}\right)$, i.e. the complexity of the original Dijkstra algorithm. We have demonstrated the use of the B-Dijkstra algorithm in ATM networks for various traffic types, using recent research results regarding congestion control of $A B R$ traffic and scheduling and traffic shaping of VBR traffic.

Simulation experiments based on homogeneous scenarios illustrate the concepts involved in routing end-to-end QOS constrained traffic. Although the overall behavior of the proposed routing algorithms is well described by the simulation results, the system under study was highly dynamic due to allocation and deallocation of resources, link state broadcasting, and propagation delays. Thus, a more comprehensive set of simulations would be useful to understand how much stale link information affects the performance of QOS routing algorithms. Also, it would be important to investigate whether it pays off to have different routing strategies for different applications in a network with heterogeneous traffic.

The algorithm implementation is assumed to be decentralized (or link state). That is, each source has a global picture of the network. This is in accord with PNNI Draft Specification (1994). However, routes are computed at call arrival instants. In case route computation time is large for a particular call admission procedure, caching routes for

\footnotetext{
$\overline{{ }^{*} \text { This phenomenum is more accentuated for light network loads, when more connections are being placed. }}$
} 
various traffic classes may be an alternative. This, however, may aggravate the "stale" routing problem. How this scheme affects resource reservation will be subject for future research.

Finally, the routing of VBR and ABR traffic for multicast connections is an issue of increasing importance given that many ATM applications are of multicast nature. We are currently investigating schemes for routing one-to-many, as well as many-to-many cannections with QOS constraints.

\section{REFERENCES}

Georgiadis, L., Guerin, R. and Peris, V. (1996) Efficient Network QoS Provisioning Based on per Node Traffic Shaping. INFOCOM96, vol.1, 102-110.

Ling, T. and Shroff, N. (1996) Scheduling Real-Time Traffic in ATM Networks. INFOCOM96, vol.1, 198-205.

Mascolo, S., Cavendish, D. and Gerla, M. (1996) ATM Rate Based Congestion Control Using a Smith Predictor: an EPRCA Implementation. INFOCOM'96, vol.2, 569-576.

Wang, Z. and Crowcroft, J. (1995) Bandwidth-Delay Based Routing Algorithms. GLOBECOM'95, 2129-2133.

Various contributors (1994) PNNI Draft Specification. ATM Forum 94-0471R9.

Parekh, A. K. and Gallager, R. (1994) A Generalized Processor Sharing Approach to Flow Control in Integrated Services Networks: The Multiple Node Case. IEEE/ACM Transactions on Networking, Vol. 2, No. 2, 137-150.

Parekh, A. K. and Gallager, R. (1993) A Generalized Processor Sharing Approach to Flow Control in Integrated Services Networks: The Single-Node Case. IEEE/ACM Transactions on Networking, Vol. 1, No. 3, 344-357.

Hassin, R. (1992) Approximation Schemes for the Restricted Shortest Path Problem. Mathematics of Operations Research, vol. 17, no.1, 36-42.

Cormen, Leiserson and Rivest (1990) Introduction to Algorithms, McGraw-Hill.

\section{ACKNOWLEDGEMENT}

This work has been supported by CNPq Grant 201597/93-4 and by NSF Grant NCR-9305376 


\section{BIOGRAPHY}

Dirceu Cavendish received his B. S. degree in electronics from Federal University of Pernambuco, Brazil, in 1986. From 1986 to 1991 he worked for telecommunication division of Philips, Brazil. From 1991 to 1994 he was an exchange student, supported by Mombusho, the Ministry of Education, in Japan. He received his M. S. degree in Computer Science from Kyushu Institute of Technology in 1994. Since then he has joined the Computer Science Department of the University of California, Los Angeles, where he is currently a graduate student of the computer networks Ph.D. program. His research interests includes congestion control and routing in high speed networks.

Mario Gerla was born in Milan, Italy. He received a graduate degree in engineering from the Politecnico di Milano, in 1966, and the M.S. and Ph.D. degrees in engineering from UCLA in 1970 and 1973, respectively. He joined the Faculty of the UCLA Computer Science Department in 1977. His research interests cover the performance evaluation, design and control of distributed computer communication systems and high speed computer networks (B-ISDN and Optical Networks). 